\title{
Crystal structure of papain-E64-c complex
}

\author{
Binding diversity of E64-c to papain $S_{2}$ and $S_{3}$ subsites
}

\author{
Moon-Jib KIM, ${ }^{*} \dagger$ Daisuke YAMAMOTO, ${ }^{*} \ddagger$ Keita MATSUMOTO,* Masatoshi INOUE,* Toshimasa ISHIDA, ${ }^{*} \S$ \\ Hiroshi MIZUNO, $\|$ Shigeyuki SUMIYA $\rrbracket$ and Kunihiro KITAMURA $\|$ \\ *Department of Physical Chemistry, Osaka University of Pharmaceutical Sciences, 2-10-65 Kawai, Matsubara, Osaka 580, Japan, \\ \|Department of Molecular Biology, National Institute of Agrobiological Resources, Tsukuba, Ibaraki 305, Japan, \\ and ๆResearch Center, Taisho Pharmaceutical Co. Ltd., 1-403 Yoshino-cho, Ohmiya, Saitama 330, Japan
}

\begin{abstract}
In order to investigate the binding mode of E64-c (a synthetic cysteine proteinase inhibitor) to papain at the atomic level, the crystal structure of the complex was analysed by X-ray diffraction at $1.9 \AA$ ( $1 \AA$ is expressed in SI units as $0.1 \mathrm{~nm}$ ) resolution. The crystal has a space group $P 22_{1} 2_{1} 2_{1}$ with $a=43.37, b=102.34$ and $c=49.95 \AA$. A total of 21135 observed reflections were collected from the same crystal, and 14811 unique reflections of up to $1.9 \AA$ resolution [Fo $>3 \sigma(F o)]$ were used for the structure solution and refinement. The papain structure was determined by means of the molecular replacement method, and then the inhibitor was observed on a $(2|F o|-|F c|)$ difference Fourier map. The complex structure was finally refined to $R=19.4 \%$ including 207 solvent molecules. Although this complex crystal (Form II) was polymorphous as compared with the previously analysed one (Form I), the binding modes of leucine and isoamylamide moieties of E64-c were significantly different from each other. By the calculation of accessible surface area for each complex atom, these two different binding modes were both shown to be tight enough to prevent the access of solvent molecules to the papain active site. With respect to the E64-c-papain binding mode, molecular-dynamics simulations proposed two kinds of stationary states which were derived from the crystal structures of Forms I and II. One of these, which corresponds to the binding mode simulated from Form I, was essentially the same as that observed in the crystal structure, and the other was somewhat different from the crystal structure of Form II, especially with respect to the binding of the isoamylamide moiety with the papain $\mathrm{S}$ subsites. The substrate specificity for the papain active site is discussed on the basis of the present results.
\end{abstract}

\section{INTRODUCTION}

Cysteine proteinases with highly reactive cysteine residues at the active site are abundant within living cells and play important roles in intracellular proteolysis (Barrett, 1977; Kirschke \& Barrett, 1987; Katunuma, 1989). Abnormal elevation of proteolytic activity is associated with serious diseases such as muscular dystrophy (Katunuma \& Kominami, 1987), osteoporosis (Delaisse et al., 1984), pulmonary emphysema (Harris et al., 1975) and tumour invasion (Denhardt et al., 1987). Therefore the development of inhibitors which can modify proteolytic activity has become an important challenge in drug design.

E64-c $\{N-[N$-(L-3-trans-carboxyoxirane-2-carbonyl)-L-leucyl]isoamylamide $\}$ is a potent inhibitor developed from the natural compound E64 (Hanada et al., 1978a,b) and inactivates most cysteine proteinases irreversibly by forming a covalent bond between the E64-c epoxy C-2 atom and the active cysteine $\mathrm{S}^{\gamma} \mathrm{H}$ group of these proteinases (Yabe et al., 1988; Matsumoto et al., 1989). In order to investigate the mechanism of the inhibition of cysteine proteinases at the atomic level, we previously analysed the crystal structure of papain (a cysteine proteinase)-E64-c complex at $2.1 \AA(0.21 \mathrm{~nm})$ resolution by X-ray diffraction and discussed a common binding mode for the E64 family (Yamamoto et al., 1991). Recently, we succeeded in preparing another type of crystal of papain-E64-c complex (polymorph) under the same crystallization conditions as the earlier one. Since it is important to know all possible binding modes of E64-c to papain in order to understand the inhibitory mechanism more exactly, this report deals with the X-ray structure analysis of this complex crystal (hereafter, the previous and current complex crystals are designated Forms I and II respectively). The chemical formulae of E64 and E64-c are given in Fig. 1, where the structure of ZPACK (a substrate-mimicking inhibitor) is also given for later discussion.

\section{MATERIALS AND METHODS}

\section{Crystallization}

The purification and preparation processes of the papain and E64-c molecules were the same as those described previously for the preparation of Form I complex (Yamamoto et al., 1991). Complex crystals suitable for X-ray-diffraction studies were grown by vapour diffusion. The complex was dissolved to a $1.5 \%(\mathrm{w} / \mathrm{v})$ concentration in $76 \mathrm{~mm}-\mathrm{NaCl}$ solution containing $64 \%$ methanol/ethanol $(2: 1, v / v)$ and equilibrated against $0.1 \mathrm{~m}$-aminoethanol/HCl buffer (pH 9.2) containing $64 \%$ methanol/ethanol $(2: 1, \mathrm{v} / \mathrm{v})$ solution. The crystals, grown by the sitting drop method at room temperature $\left(20^{\circ} \mathrm{C}\right)$, developed in approx. 10 days.

The crystals belong to space group $P 2_{1} 2_{1} 2_{1}$ with $a=43.37$, $b=102.34$ and $c=49.95 \AA$, and contain a complex pair in the asymmetric unit. This crystal (Form II) differs from Form I in the cell constants, especially the dimension of the $b$-axis and the distribution of X-ray-diffraction intensities. The cell constants of Form $I$ are $a=42.90, b=95.51$ and $c=49.99 \AA$. Therefore these crystals are polymorphous to each other.

Abbreviations used: MD, molecular dynamics; r.m.s., root mean square.

$\dagger$ Present address: Department of Physics, Soonchunhyang University, P.O.B. 97, Onyang, Chungnam 331, Republic of Korea.

$\ddagger$ Present address: Biomedical Computation Center, Osaka Medical College, 2-7 Daigaku-machi, Takatsuki 569, Japan.

$\S$ To whom correspondence should be addressed. 


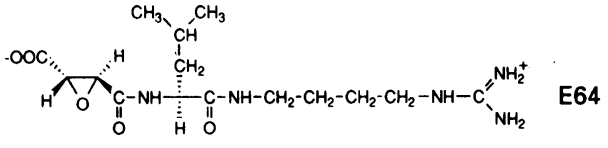

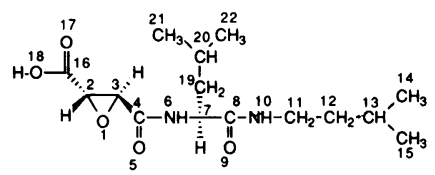

E64-c

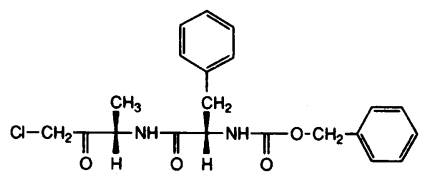

ZPACK

Fig. 1. Chemical structures of E64, E64-c and ZPACK (benzyloxycarbonyl-L-phenylalanyl-L-alanine chloromethylketone)

Atomic numbering is shown for E64-c.

\section{Data collection}

A single crystal with dimensions $0.4 \mathrm{~mm} \times 0.3 \mathrm{~mm} \times 0.2 \mathrm{~mm}^{3}$, which was sealed in a thin-walled glass capillary with a small amount of mother liquor, was mounted on a Rigaku computercontrolled four-circle diffractometer. Intensity data of 21135 observed reflections to $1.9 \AA$ resolution were collected, using $\mathrm{Ni}$ filtered $\mathrm{Cu} \mathrm{K} \alpha$ radiation and the $\omega$-scan method; the scan speed was $4{ }^{\circ} \mathrm{C} / \mathrm{min}$, and background for each reflection was measured for $5 \mathrm{~s}$. The same crystal was used for data collection throughout a period of approx. 4 days. Four intensity control reflections showed a linear decay at less than $8.0 \%$, and a decay correction was applied as a function of time. The $R_{\text {sym. }}$ of $2.4 \%$ was obtained after decay, absorption and Lp corrections of the reflection data. The scale factor and overall thermal factor by the Wilson plot were 1.12 and $14.1 \AA^{2}$ respectively; the merging $R$ factor was $8.7 \%$ for all reflections.

\section{Structure solution}

The structure was solved by use of the molecular-replacement technique with the program package MERLOT (Fitzgerald, 1988). The atomic co-ordinates of papain in the papain-ZPACK complex crystal (Drenth et al., 1976) were used as a search structure. The search structure was put into an artificial unit cell with all dimensions equal to $100.0 \AA$ and all angles equal to $90.0^{\circ}$. Using reflection data between $8.0 \AA$ and $4.0 \AA$ resolution with $I>\sigma(I)$, we calculated a full-rotation function map, where the highest peak was found at Euler angles of $\alpha=87^{\circ}, \beta=27^{\circ}$ and $\gamma=170^{\circ}$; the height of the next highest peak was approximately half that of the first.

All translation space was searched by the translation function calculation for the data between 10.0 $\AA$ and 4.0 $\AA$, and a distinct maximum peak was located for the three components of the translation vector. After rigid-body optimization of rotational and translational parameters, the packing of molecules in the cell was calculated. No abnormal short contacts were obtained, indicating that these parameters are sterically reasonable.

\section{Structure refinement}

The stereochemically restrained least-squares method formulated by the PROLSQ (Hendrickson \& Konnert, 1980) system was used for structure refinement. The structure was modified using several types of Fourier calculations by the PROTEIN system (Steigemann, 1985) and the manual model revision by the FRODO program (Jones, 1978). The usual restraints were applied for the bond distances, angles, planar groups, chiral volumes, van der Waals contacts and individual temperature factors. The progress of refinement is summarized in Table 1. The Fourier map calculated after the third stage of refinement showed the electron density corresponding to the E64-c molecule, the binding mode (named model A) of which was rather different from the previously reported one (named model B) (Yamamoto et al., 1991). In order to make clear the true binding mode of E64-c to papain, these two different binding models were separately included in the refinement from the fourth to eighth stages. In the case of model A, a clear cleavage of electron-density map was observed at the centre of the isoamylamide group in the $(2|F o|-|F c|)$ map at the eighth stage, where the final $R$ value was $24.4 \%$. This was in contrast with the map of model B $(R=23.1 \%)$ showing reasonably continuous density. Furthermore, an $(|F o|-|F c|)$ map, which was calculated with phases based on model B but omitting the E64-c atoms from the model, also showed the electron-density map corresponding to the E64-c molecule similar to that shown in Fig. 4, whereas the inhibitor molecule was not continuously traced in the map calculated from model A. All of these results clearly indicated that the binding mode of E64-c to the papain was different from the previously reported one. In the refinement of complex structure from the ninth to eleventh stages, thus only model B was considered. Solvent molecules included in the structure refinement were limited to those having electron densities greater than $0.40 \mathrm{e} / \AA^{3}$ on the map. The final $R$ value at the eleventh stage was refined to 0.194 by using 14811 reflections $[I>3 \sigma(I)]$ at $1.9 \AA$ resolution.

Table 1. Summary of the stereochemically restrained least-squares refinement

Abbreviation used: R.m.s., root mean square. $1 \AA=0.1 \mathrm{~nm}$.

\begin{tabular}{|c|c|c|c|c|c|c|c|c|c|c|c|}
\hline Stage... & 1 & 2 & 3 & 4 & 5 & 6 & 7 & 8 & 9 & 10 & 11 \\
\hline No. of cycles & 5 & 9 & 6 & 7 & 9 & 9 & 9 & 14 & 10 & 15 & 6 \\
\hline Resolution (A) & $10-3$ & $4-2.4$ & $4-2.4$ & $4-2.4$ & $7-2.4$ & $7-2.4$ & $7-2.4$ & $20-1.9$ & $20-1.9$ & $20-1.9$ & $20-1.9$ \\
\hline $\mathrm{Fo} / \sigma(\mathrm{Fo})$ & 3 & 3 & 3 & 3 & 3 & 3 & 3 & 3 & 3 & 3 & 3 \\
\hline No. of reflections & 4731 & 6847 & 6847 & 6847 & 8683 & 8683 & 8683 & 148.11 & 14811 & 14811 & 14811 \\
\hline No. of parameters & 6621 & 6621 & 6621 & 6709 & 6709 & 6709 & 6877 & 7805 & 7665 & 7593 & 7537 \\
\hline $\begin{array}{l}\text { No. of atoms } \\
\text { (solvents) }\end{array}$ & 1655 & 1655 & 1655 & 1677 & 1677 & 1677 & $\begin{array}{l}1719 \\
(42)\end{array}$ & $\begin{array}{l}1951 \\
(274)\end{array}$ & $\begin{array}{l}1916 \\
(239)\end{array}$ & $\begin{array}{l}1898 \\
(221)\end{array}$ & $\begin{array}{l}1884 \\
(207)\end{array}$ \\
\hline$\langle F o-F c\rangle$ & 103.1 & 186.8 & 161.1 & 143.1 & 78.83 & 66.58 & 61.51 & 50.77 & 43.88 & 42.56 & 41.68 \\
\hline R.m.s. shifts & 0.208 & 0.277 & 0.419 & 0.478 & 0.384 & 0.201 & 0.138 & 0.270 & 0.229 & 0.123 & 0.049 \\
\hline$R$ (initial) $(\%)$ & 39.7 & 47.6 & 40.4 & 35.8 & 39.6 & 29.4 & 25.0 & 25.1 & 23.4 & 21.2 & 19.6 \\
\hline$R($ final $)(\%)$ & 32.1 & 38.6 & 33.3 & 29.6 & 29.3 & 24.8 & 22.9 & 23.1 & 20.5 & 19.2 & 19.4 \\
\hline
\end{tabular}



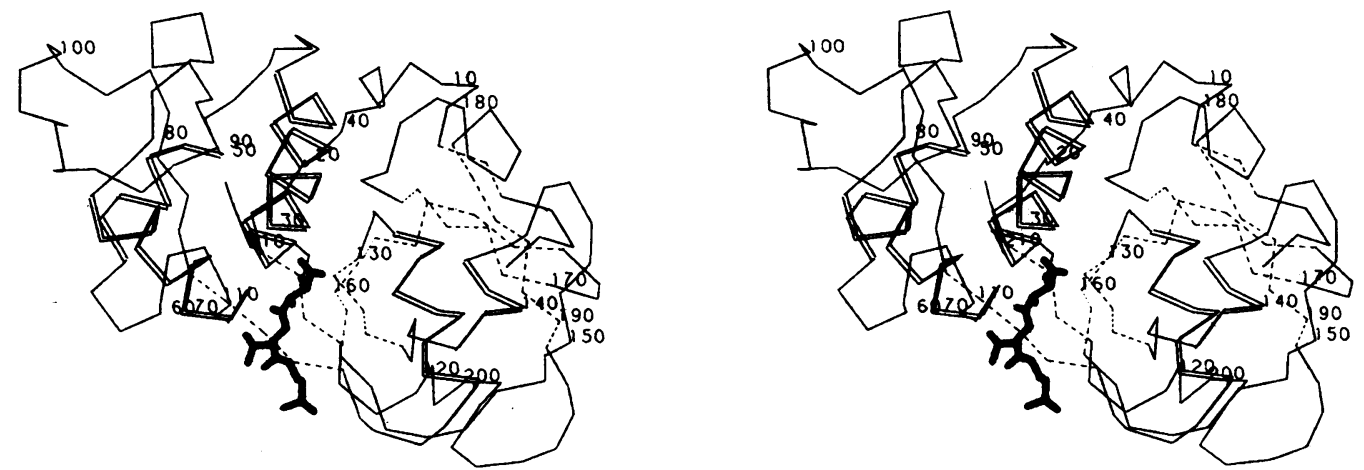

Fig. 2. Stereoscopic view of the overall structure of the papain-E64-c complex

The $\mathrm{C}^{\alpha}$ atoms of papain and E64-c molecules are shown. Helical and sheet regions of papain are presented by double-solid and single-dashed lines, respectively, and the bold solid lines represent the E64-c moiety. Residue numbers of papain are labelled at intervals of ten residues.

\section{Molecular-dynamics (MD) simulation}

Energy-minimization and MD simulations were performed with AMBER3.0 (Singh et al., 1986) using the atomic coordinates of Forms I and II with the AMBER/united atom energy parameters. Binding structures, which consisted of amino acid residues within $15 \AA$ of each atom of the E64-c molecule, were energy-minimized by the conjugate gradient method until each gradient was smaller than $42 \mathrm{~kJ} / \mathrm{mol}$ per $\AA$, at which point the distance-dependent dielectric constant of $\epsilon=R_{i \mathrm{j}}$ and the $8 \AA$ non-bonded cutoff were employed. To each of the energyminimized complexes, 411 water molecules with TIP3P energy parameters (Jorgensen et al., 1983) were then added within the sphere of an $18 \AA$ radius of each atom of the E64-c molecule. After optimization of these water molecules, they were subjected to the MD calculations during 2 ps at $310 \mathrm{~K}$ and were restrained by a boundary force of $6.3 \mathrm{~kJ} / \mathrm{mol}$ per $\AA$ to prevent their leakage from the molecular system.

The MD simulations were carried out with time steps of $0.002 \mathrm{ps}, \epsilon=1$ and a non-bonded cutoff of $8 \AA$. Initial velocities for respective molecular systems were derived from Boltzmann's distribution of $10 \mathrm{~K}$. The temperature of the respective systems,

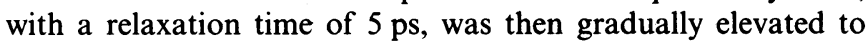
$310 \mathrm{~K}$. The successive MD simulations during $40 \mathrm{ps}$ were then calculated. In order to save computational time, the SHAKE method (van Gunsteren \& Berendsen, 1977) was used for hydrogen atoms within an $18 \AA$ sphere, and all atoms out of the sphere were fixed.

All numerical calculations were carried out on a MicroVAX II, VAX-station 3100 and Silicon graphics IRIS 2400 Turbo interactive computer graphics system at the Computation Center of Osaka University of Pharmaceutical Sciences and on a Fujitsu S-4/330CXP workstation at Biomedical Computation Center of Osaka Medical College. The atomic co-ordinates of the present complex crystal (Form II) have been deposited in the Brookhaven Protein Data Bank, from which copies are available.

\section{RESULTS AND DISCUSSION}

\section{Overall structure of complex crystal}

The complex structure of papain and E64-c is shown in Fig. 2. Papain has 212 amino acid residues and is folded into two domains: an L-domain consisting of residues 10-11 and 207-212 and an R-domain consisting of the remaining residues. The Cys25 active centre of papain is positioned at the entrance of the $\alpha$ helix (residues 24-42) in the L-domain. The detailed X-ray

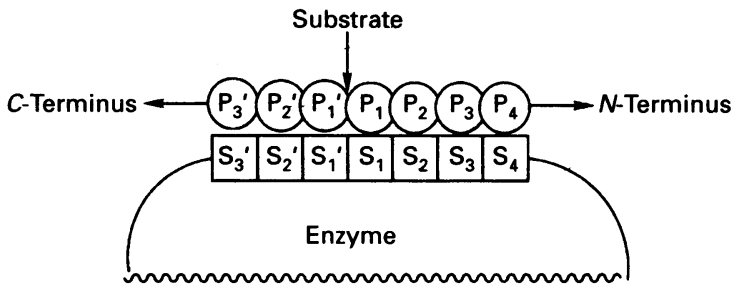

Fig. 3. Description of the papain active site according to the concept of Schechter \& Berger (1967)

The residues of the papain and substrate are denoted by $\mathbf{S}$ and $\mathbf{P}$ respectively. The arrow indicates the scissile bond.

analysis of papain at $1.65 \AA$ resolution has already been performed (Kamphuis et al., 1984) and showed no significant difference from our measurements concerning the backbone chain conformation. Most of the conformational $\phi / \psi$ torsion angles are found within the outer limit boundary of the Ramachandran plot (Ramakrishnan \& Ramachandran, 1965) and the secondary structure of papain is little affected by the binding of E64-c.

With respect to the papain crystal, six kinds of polymorphs have been reported (Forms A, B, C, D, E and S) (Drenth et al., 1971). Of these, the crystal structures of Forms $C$ and D (Drenth et al., 1976; Kamphuis et al., 1984; Priestle et al., 1984) have been analysed. The difference between Form $C$ and $D$ can be characterized by (a) the different $b$-axis (Form $C$ is longer than D) and (b) the different orientation of papain molecules with respect to the unit cell axis, although both have the same space group of $P 2_{1} 2_{1} 2_{1}$. The current complex crystal of Form II could be judged to belong to Form $C$, whereas the earlier Form I complex belongs to Form D. Therefore it is obvious that Forms I and II are polymorphous to each other.

The active site of papain was defined according to the concepts of Schechter \& Berger (1967), as illustrated in Fig. 3, in which the $\mathrm{S}$ subsites $\left(\mathrm{S}_{1}-\mathrm{S}_{4}\right)$ of papain interact with four peptidyl substrate residues $\left(\mathrm{P}_{1}-\mathrm{P}_{4}\right)$ of the $N$-terminal side of the scissile bond, and $\mathrm{S}^{\prime}$ subsites $\left(\mathrm{S}_{1}{ }^{\prime}-\mathrm{S}_{3}{ }^{\prime}\right)$ interact with three residues $\left(\mathrm{P}_{1}{ }^{\prime}-\mathrm{P}_{3}{ }^{\prime}\right)$ of the substrate $C$-terminal side. The E64-c inhibitor in Form II is located at the groove created between the L- and R-domains and binds along the $S$ subsites $\left(S_{1}-S_{3}\right)$ of papain. The binding of the inhibitor to the $S$ subsites has also been observed in Form I and in the complex crystal of papain-E64 (Varughese et al., 1989), and is very similar to that of ZPACK, a papain substrate analogue (Drenth et al., 1976). Contrary to earlier predictions 


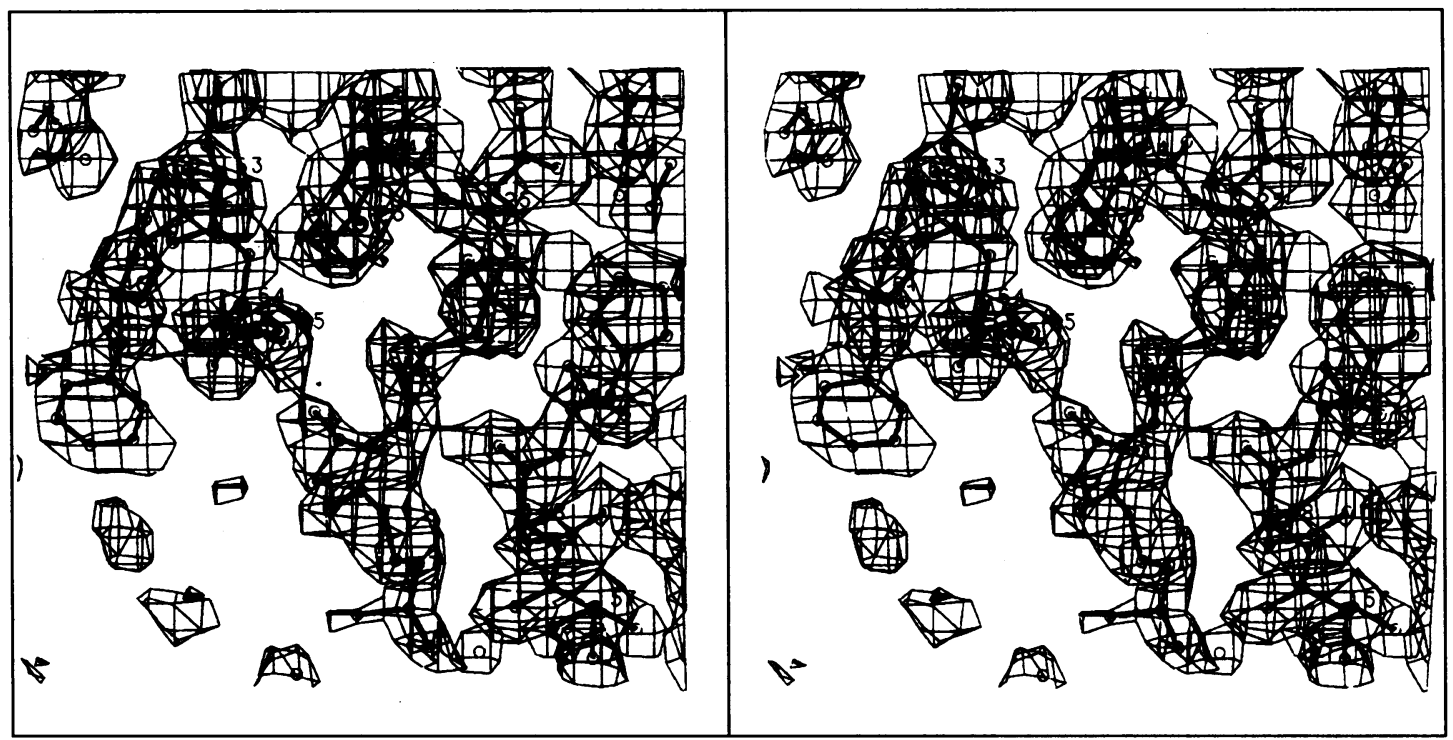

Fig. 4. Stereoscopic electron-density map of the E64-c binding site at the final stage of refinement

Electron density of the E64-c moiety, positioned at the centre, binds to that of the Cys-25 $\mathrm{S}^{\gamma}$ atom via the epoxy C-2 atom. This map is clearly distinguished from those of neighbouring residues such as Gln-19, His-159, Asp-158, Val-157, Tyr-61 and Gly-66.
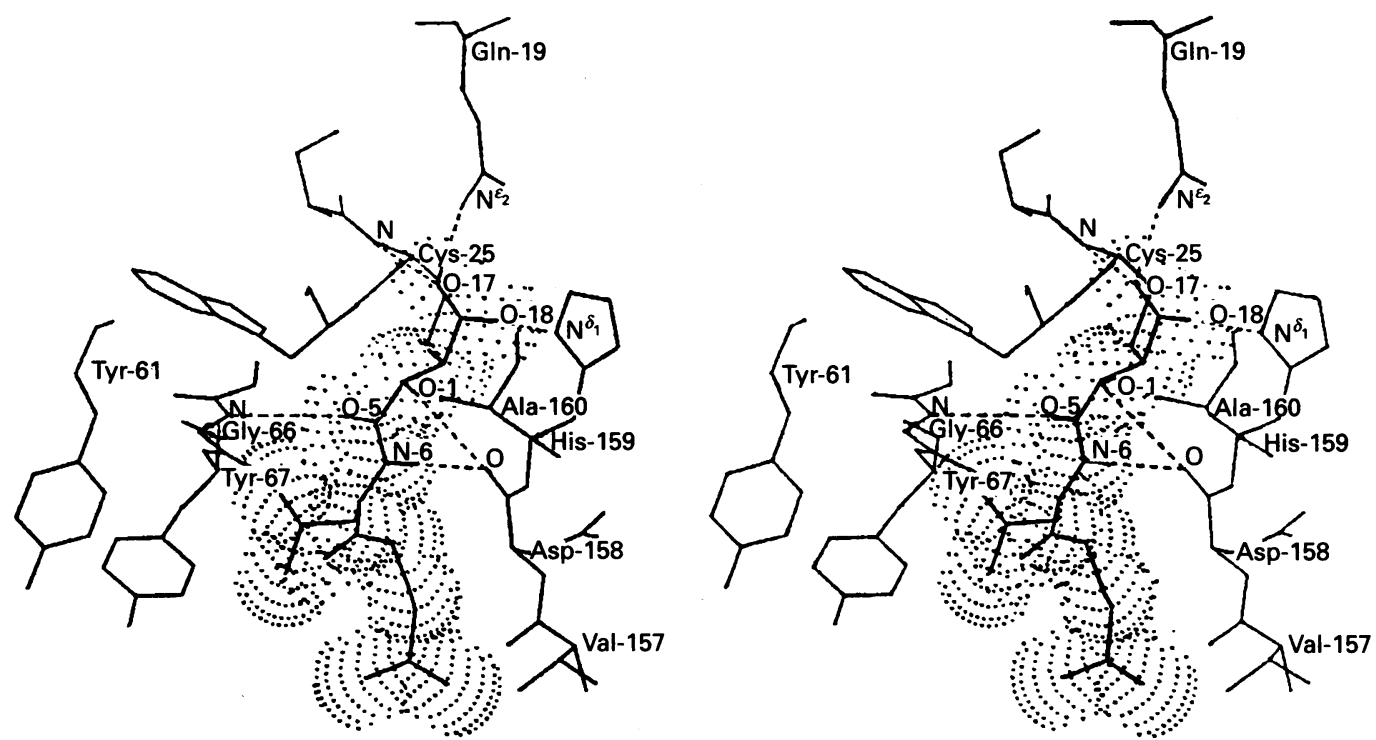

Fig. 5. Stereoscopic drawing of the E64-c binding site

The structure of E64-c is distinguished with the dotted van der Waals surface, and possible hydrogen bonds are also shown by broken lines.

(Barrett et al., 1982; Rich, 1986), this means that the main chains of substrate and inhibitor both interact with the same $\mathrm{S}$ subsites of papain.

\section{Structure of the E64-c binding site}

The electron-density map of the E64-c binding site is shown in Fig. 4, which is a $(2|F o|-|F c|)$ map calculated with phases based on the complex structure at the final stage of refinement but omitting the inhibitor atoms. E64-c is bound to the Cys-25 with a covalent bond formed between the epoxy C-2 atom of E64-c and the Cys-25 $\mathrm{S}^{\gamma} \mathrm{H}$ group of papain. The consequent E64-c adduct has an $R$ configuration at $\mathrm{C}-2$, and forms a free $\mathrm{OH}$ group at the C-3 atom. Such covalent bond formation is also seen in Form I. The significant interactions between the E64-c and the papain active site are summarized in Fig. 5 and Table 2. The
E64-c molecule is linked by four hydrogen bonds and three short contacts with the catalytic site (Gln-19, Cys-25 and His-159) and S subsite (Gly-66 and Asp-158) of papain; thus the L-transsuccinyl moiety of the inhibitor is tightly held by these interactions. This moiety of E64-c has low thermal factors similar to those of the main-chain atoms of helical and sheet regions in papain. Each of two oxygen atoms (O-17 and O-18) and the E64-c terminal carboxy group participates bifurcately in hydrogen bonds or short contacts with the polar atoms of Gln19, Cys-25 and His-159. The hydroxy O-1 atom, formed by the covalent bond between the E64-c epoxy ring and the Cys-25 S ${ }^{\gamma} \mathrm{H}$ group, is located in the vicinity of the Asp-158 O (main chain), and a weak hydrogen bond is possible between them. The $0-5$ and N-6 atoms of the inhibitor peptide participate in the hydrogen bonds with Gly-66 N and Asp-158 O atoms of papain, though 
Table 2. Interactions between E64-c and papain active site

$1 \AA=0.1 \mathrm{~nm}$.

\begin{tabular}{lllc}
\hline $\begin{array}{l}\text { Type of } \\
\text { bond }\end{array}$ & Atom & Residue & Length (Å) \\
\hline Covalent bond & C-2 & Cys-25 S & 1.80 \\
Hydrogen bonds & N-6 & Asp-158 O & 2.97 \\
& O-17 & Gln-19 N & 2.46 \\
& O-17 & Cys-25 N & 3.09 \\
& O-18 & His-159 N & 2.94 \\
Short contacts & O-1 & Asp-158 O & 3.39 \\
& O-5 & Gly-66 N & 3.56 \\
& O-18 & Gln-19 N & 3.48
\end{tabular}

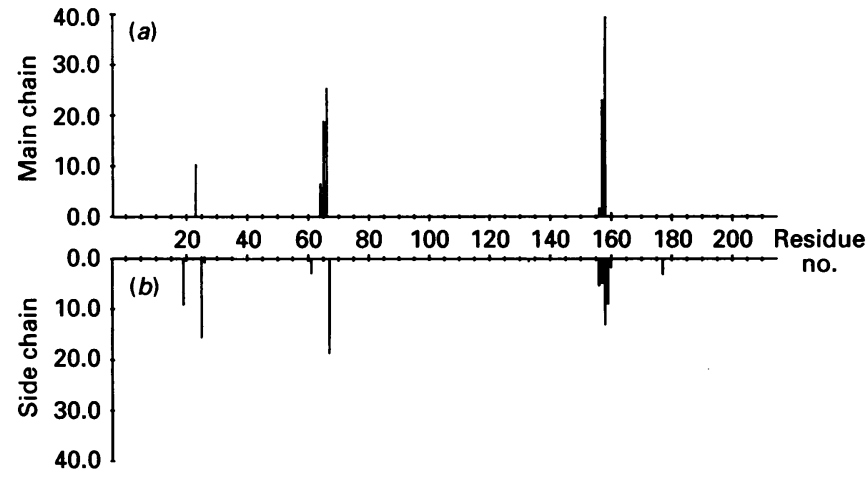

Fig. 6. Difference of accessible surface area (DASA) $\left(\AA^{2}\right)$ of each mainchain $(a)$ and side-chain $(b)$ atom

the former interaction is fairly weak. As in the case of Form I, no direct participation of solvent molecules is observed for the interaction between the E64-c and papain in Form II.

The leucyl side chain and isoamylamide moiety of E64-c were held by hydrophobic interactions (van der Waals contacts) with the residues of Gly-66 and Tyr-67 and of Val-157 and Asp-158 respectively. These hydrophobic groups of the inhibitor are located at the entry of the hydrophobic pocket corresponding to the $S_{2}$ and $S_{3}$ subsites of papain.

In order to estimate the binding fitness of E64-c to papain, the

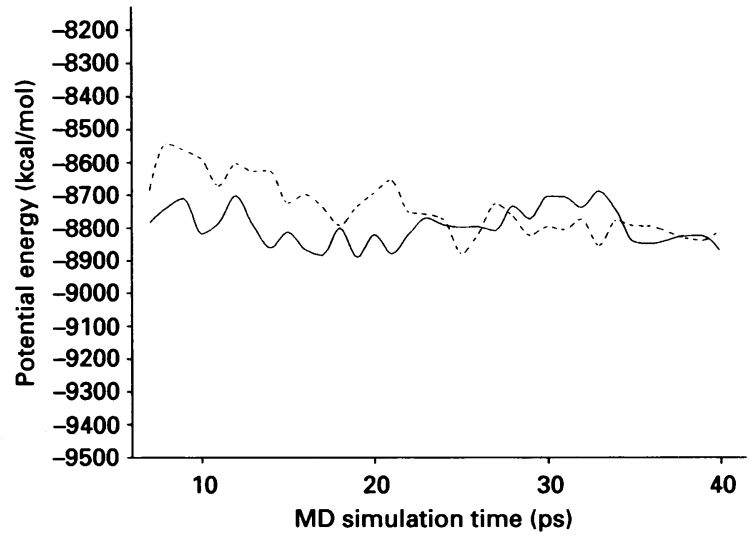

Fig. 8. Variations of potential energies (in $\mathrm{kJ} / \mathrm{mol}$ ) as a function of $\mathrm{MD}$ simulation time (in ps) in Forms I (-) and II (----).

$1 \mathrm{kcal}$ is equivalent to $4.2 \mathrm{~kJ}$.

difference of accessible surface area (DASA) was calculated using

$$
\text { DASA = ASA (enzyme)-ASA (complex) }
$$

where ASA represents the accessible surface area given by Lee $\&$ Richard (1971), and ASA (enzyme) and ASA (complex) are the accessible surface area of each residue in the papain structure without and with the E64-c inhibitor respectively. The result is given in Fig. 6, and shows that each residue interacting with the inhibitor, especially Gly-66, Tyr-67, Val-157 and Asp-158, loses a significant area of its main and/or side chain accessibility to solvents. This means that the $S$ subsites of papain are almost shielded from the solvent phase by binding with the E64-c molecule, and this interpretation is also suggested by the low thermal factors of E64-c chains as compared with those of papain surface atoms.

\section{Binding variation of E64-c for the papain active site}

In order to show the binding differences of E64-c Form I and Form II for the papain active site, both binding modes were superimposed so that the root mean square deviation between the corresponding atoms was minimized (Fig. 7). In contrast with the well-conserved disposition of the papain active site, the binding modes of each inhibitor differ from each other. Although
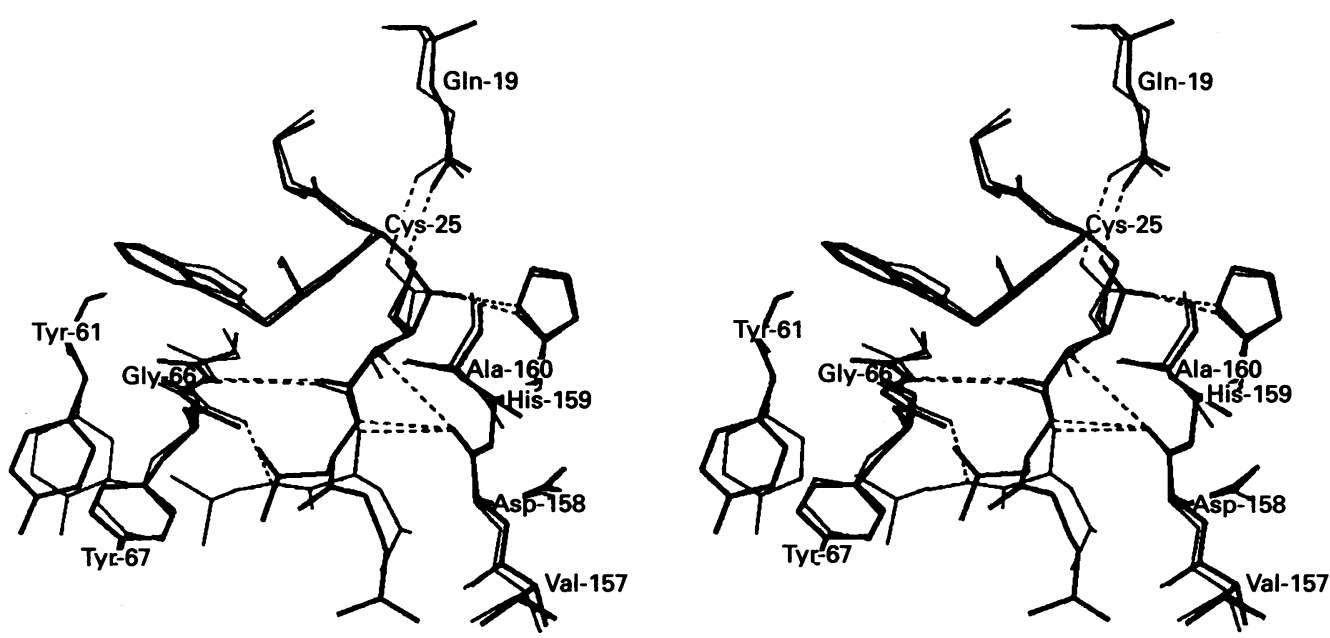

Fig. 7. Stereoscopic view of the superimposition between the E64-c molecules in the crystal structures of Forms I and II

The papain active site and E64-c are shown by thin and thick lines for Forms I and II respectively. 

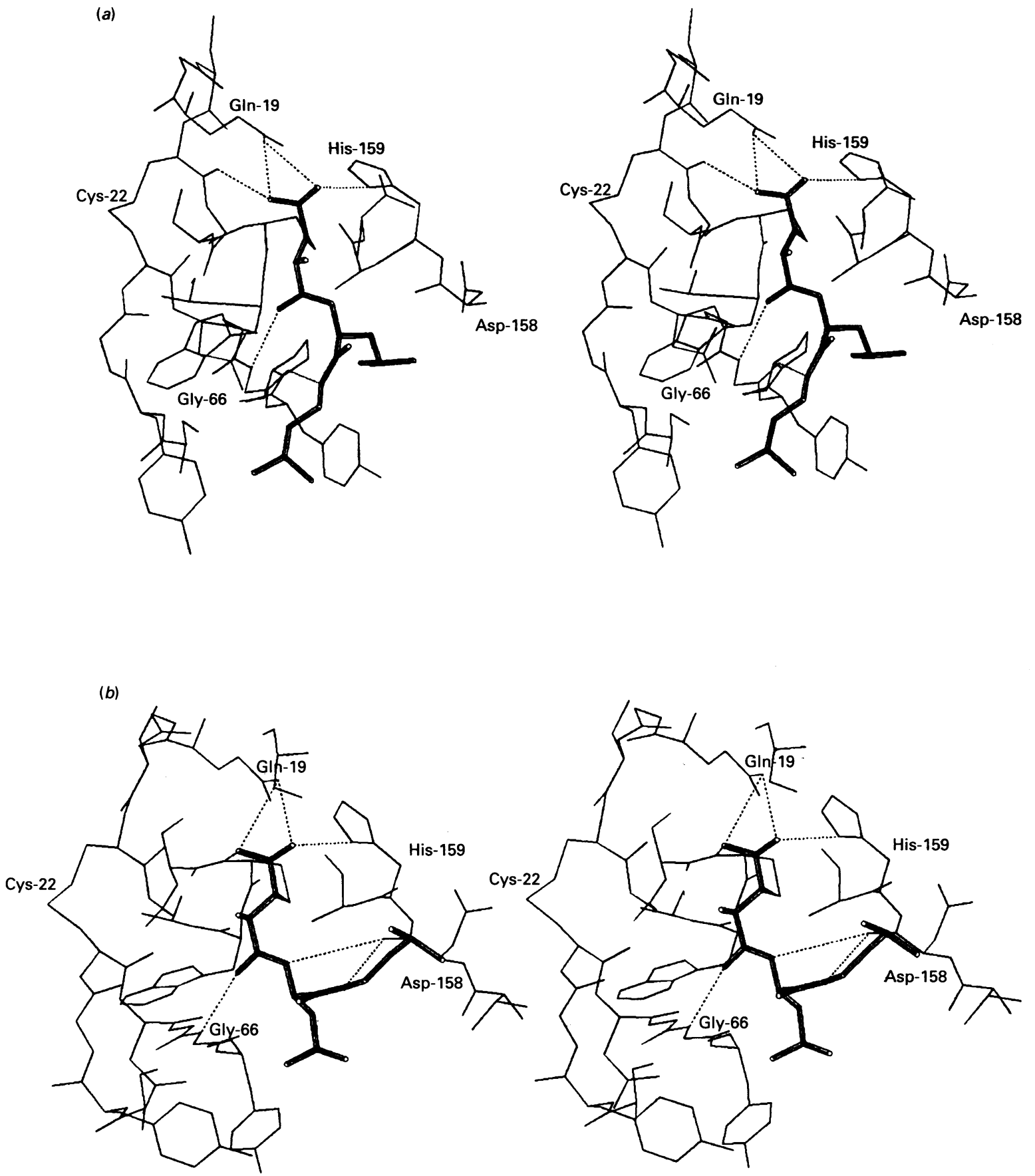

Fig. 9. Stereoscopic snapshots of E64-c binding to papain at $40 \mathrm{ps}$ simulated from the complex structures of Forms I (a) and II (b)

The dotted lines represent possible hydrogen bonds. E64-c is shown by the thick lines.

the hydrogen bonds and electrostatic short contacts in which the succinyl carboxy moiety of the inhibitor participates are nearly the same, the orientations of the leucyl side chain and isoamylamide chain are almost opposite in each form, as reflected by the significant differences of C-4-N-6-C-7-C-8 and N-6C-7-C-8-N-10 torsion angles: $-43.9^{\circ}$ and $67.3^{\circ}$ for Form $\mathrm{I}$ and $-165.7^{\circ}$ and $-10.3^{\circ}$ for Form II. These binding differences of the two forms of E64-c are probably due to the lack of polar atoms in its terminal chain. In the E64-papain complex (Varughese et al., 1989), the guanidyl terminal of E64 was hydrogen-bonded to the Tyr-61 and Tyr-67 OH groups, and consequently there was no chance to cause such a binding variation.
In order to determine whether Form I or II is preferable for the binding of E64-c, both complexes were subjected to MD simulations. The variations of respective potential energies were monitored as a function of simulation time, as shown in Fig. 8. The averaged r.m.s. difference between the potential energies of Forms I and II during $21-40$ ps MD simulation corresponds to $1.38 \mathrm{~kJ}$ per residue and means that the energy difference between Forms I and II is not significant. The fluctuation ratios of Forms I and II against their total potential energies were both less than $1 \%$, far below the commonly accepted $5 \%$ value for the protein conformation in the stationary state (van Gunsteren \& Berendsen, 1977). Thus the conformations of Forms I and II simulated for longer than $21 \mathrm{ps}$ are in stationary states with 
nearly the same energies. The snapshots taken at $40 \mathrm{ps}$ of the MD simulations reflect representative features of the binding modes (hydrogen bonds and short contacts) commonly observed during the simulation period of $21-40$ ps (Fig. 9). The binding mode of (Fig. 9a) of E64-c derived from Form I was little changed during the MD simulation and was nearly the same as that observed in the crystal structure. On the other hand, the binding mode in Fig. $9(b)$, derived from Form II, showed a somowhat different conformation from the starting structure of E64-c, especially for the isoamylamide moiety, although the energy variations for respective MD conformers were small. This binding change is mainly due to the hydrogen-bond formation between the $\mathrm{N}-10$ (E64-c) and $\mathrm{O}$ (Asp-158 in papain) atoms, thus leading to the parallel alignment between the isoamylamide moiety of E64-c and the side chain of Asp-158. In conclusion, the MD simulation covering 40 ps demonstrates that Forms I and II are essentially in stable stationary states and no significant energetic difference exists between the two different binding modes.

\section{Biochemical implication of the complex structure}

In general, it is well recognized that the interaction between the subsites of a proteinase and the amino acid residues of the peptide substrate can significantly contribute to enhancement of catalytic activity. Early studies on the substrate specificity of papain (Berger \& Schechter, 1970) indicated that the $S_{2}$ subsite governs the specificity by accepting a hydrophobic amino acid residue from the $\mathrm{P}_{2}$ position of the substrate (see Fig. 3). In particular, a high specificity for $P_{2}=$ Phe was identified at the $S_{2}$ site. In the crystal structures of papain-E64 (Varughese et al., 1989) and papain-E64-c Form I (Yamamoto et al., 1991) complexes, the leucine residue of inhibitor corresponds to the $\mathbf{P}_{2}$ site, and it is accepted by the papain $\mathrm{S}_{2}$ subsite consisting of $\mathrm{Val}-$ 133, Val-157 and Asp-158. A similar binding pattern is also observed in the crystal structure of the papain-ZPACK complex (Drenth et al., 1976), thus following the general concept concerning substrate specificity of the proteinase.

On the other hand, the present X-ray crystal analysis of papain-E64-c Form II complex shows another type of binding mode; i.e. the isoamylamide group, which is supposed to correspond to the $P_{3}$ site of the substrate, is locked by the $S_{2}$ subsite, and the leucyl side chain is instead held by van der Waals contacts with the $S_{3}$ subsite consisting of papain Gly-66 and Tyr67. Since the binding modes of E64-c in Forms I and II are both tightly locked at the papain $S$ subsites in such a way that the solvent molecules are completely shielded and are in energetically stable stationary states, we propose that the binding site of papain should not simply be divided into $S_{2}$ and $S_{3}$ independently, but rather be considered as a large hydrophobic pocket consisting of $\mathrm{S}_{2}$ and $\mathrm{S}_{3}$ subsites. As shown in Fig: 2, this large hydrophobic core is located at the entrance of the groove created by the $\mathrm{R}$ - and L-domains, and is bordered with solvent molecules. Thus, it may be unreasonable to consider the substrate specificity at the $\mathrm{P}_{n}$ site for $n>3$.

In summary, we have shown that the crystal structure of Form II differs from its polymorph Form I in several respects. Among them, the most significant difference is that an alternative conformation of E64-c is observed in which the $P_{2}$ and $P_{3}$ residues are swapped. This information means that some inhibitors may bind in multiple modes, a fact that clearly is of interest in understanding enzyme mechanisms as well as structurebased drug design.

\section{REFERENCES}

Barrett, A. J. (1977) Proteinases in Mammalian Cells and Tissue, Elsevier/North-Holland, Amsterdam

Barrett, A. J., Kembhavi, A. A., Brown, M. A., Kirschke, H., Knight, C. G., Tamai, M. \& Hanada, K. (1982) Biochem. J. 201, 189-198

Berger, A. \& Schechter, I. (1970) Philos. Trans. R. Soc. London, Ser. B 257, 249-264

Delaisse, J. M., Eeckhout, Y. \& Vaes, G. (1984) Biochem. Biophys. Res. Commun. 125, 441-447

Denhardt, D., Greenberg, A. H., Egan, S. E., Hamilton, R. T. \& Wright, J. A. (1987) Oncogene 2, 55-59

Drenth, J., Jansonius, J. N., Koekoek, R. \& Wolthers, B. G. (1971) Adv. Protein Chem. 25, 79-115

Drenth, J., Kalk, K. H. \& Swen, H. M. (1976) Biochemistry 15, 3731-3738

Fitzgerald, P. M. D. (1988) J. Appl. Crystallogr. 21, 273-278

Hanada, K., Tamai, M., Ohmura, S., Sawada, J., Seki, T. \& Tanaka, I. (1978a) Agric. Biol. Chem. 42, 529-536

Hanada, K., Tamai, M., Yamagishi, M., Ohmura, S., Sawada, J. \& Tanaka, I. (1978b) Agric. Biol. Chem. 42, 523-528

Harris, J. O., Olson, G. N., Castle, J. R. \& Maloney, A. S. (1975) Am. Rev. Respir. Dis. 111, 579-586

Hendrickson, W. A. \& Konnert, J. H. (1980) in Biomolecular Structure, Function, Conformation and Evolution (Srinivasan, R., ed.), vol. 1, pp. 43-57, Pergamon, Oxford

Jones, T. A. (1978) J. Appl. Crystallogr. 11, 268-272

Jorgensen, W. L., Chandrasekhar, J., Madura, J. D., Impey, R. W. \& Klein, M. L. (1983) J. Chem. Phys. 79, 926-935

Kamphuis, I. G., Kalk, K. H., Swarte, M. B. A. \& Drenth, J. (1984) J. Mol. Biol. 179, 233-256

Katunuma, N. (1989) in RBC: Cell Biology Reviews (Knecht, E. \& Grisolia, S., eds.), vol. 20, pp. 35-61, Springer-Verlag, Berlin

Katunuma, N. \& Kominami, E. (1987) Rev. Physiol. Biochem. Pharmacol. 108, 1-20

Kirschke, H. \& Barrett, A. J. (1987) in Lysosomes: Their Role in Protein Breakdown (Glaumann, H. \& Ballard, F. J., eds.), pp. 193-283, Academic Press, London

Lee, B. \& Richard, F. M. (1971) J. Mol. Biol. 55, 379-400

Matsumoto, K., Yamamoto, D., Ohishi, H., Tomoo, K., Ishida, T., Inoue, M., Sadatome, T., Kitamura, K. \& Mizuno, H. (1989) FEBS Lett. 245, 177-180

Priestle, J. P., Ford, G. C., Glor, M., Mehler, E. L., Smit, J. D. G., Thaller, C. \& Jansonius, J. N. (1984) Acta Crystallogr. A40, C17

Ramakrishnan, C. \& Ramachandran, G. N. (1965) Biophys. J. 5, 909-933

Rich, D. H. (1986) in Protease Inhibitors (Barrett, A. J. \& Salvesen, G. S., eds.), pp. 153-176, Elsevier, Amsterdam

Schechter, I. \& Berger, A. (1967) Biochem. Biophys. Res. Commun. 27, $157-162$

Singh, U. C., Weiner, P. K., Caldewell, J. W. \& Kollman, P. A. (1986) AMBER3.0, Department of Pharmaceutical Chemistry, University of California, San Francisco

Steigemann, W. (1985) A Program System for the Crystal Structure Analysis for Proteins, Max-Planck-Institut fur Biochemie, Munchen

van Gunsteren, W. F. \& Berendsen, H. J. C. (1977) Mol. Phys. 34, $1311-1327$

Varughese, K. I., Armed, F. R., Cary, P. R., Hasnain, S., Huber, C. P. \& Storer, A. C. (1989) Biochemistry, 28, 1330-1332

Yabe, Y., Guillaume, D. \& Rich, D. H. (1988) J. Am. Chem. Soc. 110, 4043-4044

Yamamoto, D., Matsumoto, K., Ohishi, H., Ishida, T., Inoue, M., Kitamura, K. \& Mizuno, H. (1991) J. Biol. Chem. 266, 14771-14777 